\title{
Colonizzazione greca. A proposito del primo volume di un nuovo manuale
}

Nel 1982 è stato pubblicato nella Cambridge Ancient History un profilo della colonizzazione greca nel Mediterraneo, redatto da A.J. Graham, che veniva ad affiancare The Greeks Overseas di J. Boardman, di taglio archeologico e già giunto in quegli anni alla terza edizione ${ }^{1}$. In poco più di cento pagine Graham ripercorreva le vicende legate all'espansione greca oltremare, senza trascurare alcuno degli aspetti essenziali del fenomeno o dei dati materiali più importanti allora noti. In un quarto di secolo la documentazione archeologica è cresciuta enormemente e così il numero degli studiosi che si sono occupati e si occupano di questi problemi. Se a ciò si aggiunge il lento, ma proficuo annodarsi di un dialogo con gli studiosi dell'Europa dell'Est, tradizionalmente impegnati sul fronte del Mar Nero, risulta evidente che il panorama è profondamente cambiato rispetto ai primi anni ' 80 e così pure le aspettative di chi si occupa di questi argomenti. Oggi è difficilmente pensabile che un unico studioso sia in grado di dominare una materia tanto vasta e in crescita continua ed è quindi nell'ordine delle cose che si debba procedere altrimenti. Una storia generale della colonizzazione greca non può ormai che essere scritta a più mani, con tutti i vantaggi, ma anche i limiti che un'operazione del genere comporta. Il vantaggio è indubbiamente quello di poter affidare la trattazione di singole aree o di specifici argomenti a degli specialisti del settore, lo svantaggio è l'inevitabile assenza di uno sguardo unitario e la possibile mancanza di coerenza interna tra le varie sezioni. Vantaggi e svantaggi di questo tipo si riscontrano nel volume in esame che, insieme ad altri due a seguire, si propone di fornire la prima trattazione organica moderna ad ampio raggio del fenomeno coloniale ${ }^{2}$. Nella prefazione è specificato che l'opera, dedicata proprio alla memoria di A.J. Graham, verrà a costituire un manuale, il primo che sia mai stato progettato su questo argomento.

Colonizzazione, come si sa, è un termine problematico anche se ormai entrato a far parte del lessico della storia antica e dunque ineliminabile a dispetto della sua riconosciuta inadeguatezza. In quest'opera, come precisa il curatore G. Tsetskhladze

1 A.J. Graham, The Colonial Expansion of Greece, in $C A H^{2}$, III, 3 (1982), p. 83-162 e The Western Greeks, ibid., p. 163-195; il volume di J. Boardman è stato pubblicato per la prima volta nel 1964 e successivamente nel 1973 e nel 1980. E' ora alla quarta edizione (The Greeks Overseas. Their Early Colonies and Trade, London, 1999 $)$.

2 Gocha R. TseTSKhladze (Éd.), Greek Colonisation. An Account of Greek Colonies and Other Settlements Overseas. Volume 1, Leiden/Boston, Brill, 2006, 1 vol. 15,5 x $24 \mathrm{~cm}$, LXXXIII-564 p., ill. (MNemosyne SuPPLementa, 193). Prix: $186.00 €$. ISSN 0169-8958, ISBN 13: 978-90-04-12204-8, ISNB 10: 90-04-12204-4. I volumi inizialmente previsti erano due. Nelle more di stampa di questa recensione è stato pubblicato il secondo volume (di cui verrà dato conto in un prossimo numero di questa stessa rivista) ed è stato annunciato che l'opera sarà completata da un terzo volume in preparazione. 
nell'introduzione (Revisiting Ancient Greek Colonisation), è usato in senso molto ampio per indicare qualunque situazione abbia dato luogo a uno stanziamento di genti greche in territorio straniero, o a una certa distanza dal loro luogo di origine, e che risulti riconoscibile e distinguibile attraverso le fonti letterarie e/o archeologiche. Dunque città greche - quelle che sono comunemente considerate le colonie 'tipo' e per le quali si tende a usare il termine apoikia - ma non solo: anche insediamenti mixellenoi o mixobarbaroi e ancora quei particolari tipi di poleis che sono gli emporia e poi le cleruchie e altro. Il problema terminologico, con i relativi risvolti concettuali, è solo il primo di una serie di temi di ordine generale discussi nell'introduzione da G. Tsetskhladze che passa in rassegna le questioni fondamentali sulle quali è chiamato oggi a confrontarsi chi si occupa di colonizzazione greca, dalle più tradizionali (cause, cronologia, modalità di fondazione) a quelle che in tempi recenti si sono imposte con maggiore urgenza all'attenzione della comunità scientifica (tipologia e nomenclatura degli insediamenti, urbanizzazione, etnicità) a quelle sulle quali è tuttora in atto un significativo riorientamento degli studi soprattutto per effetto delle ormai onnipresenti ricerche di segno post-coloniale (rapporti Greci-comunità locali, rapporti Greci-Fenici, connettività mediterranea). L'esposizione di G. Tsetskhladze è equilibrata e nell'indicare le tendenze attuali evidenzia le novità reali senza assecondare troppo le mode. Ben poco spazio, ad esempio, è concesso alle tesi radicalmente revisioniste, e ormai molto discusse, di R. Osborne ${ }^{3}$ alle quali è riservata soltanto una nota (p. XLVII-XLVIII n. 109). A parte due sviste (p. LXIII), che si segnalano nel caso di un'eventuale ristampa (Siris e Locri non sono colonie spartane e il "porto eccellente" è certo quello di Taranto e non di Metaponto), questa introduzione offre un utile status quaestionis, che tiene conto del bagaglio che abbiamo alle spalle e dei percorsi che si aprono davanti a noi.

Dopo queste pagine, nelle quali i riferimenti bibliografici rimandano pressoché esclusivamente agli ultimi studi, il lettore si trova impreparato di fronte alla distonia che lo attende quando si inoltra nel resto del volume. Appare infatti evidente, man mano che si prosegue nella lettura, che i vari capitoli sono stati tutti redatti anni addietro. La bibliografia, il lessico e il taglio delle diverse sezioni ne denunciano la redazione negli anni '90 (si pensi ad esempio all'uso ricorrente di nozioni quali "acculturazione" o "ellenizzazione") e non sempre basta a renderle attuali l'aggiunta

3 R. Osborne, Early Greek Colonization? The Nature of Greek Settlement in the West, in N. FISHER - H. VAN WEES (Eds.), Archaic Greece: New Approaches and New Evidence, London, 1998, p. 251-269 e cf. ID., Greece in the Making, 1200-479 BC, London/New-York, 1996, p. 8- 15; 114-129, 197-207; ID., Greek History, London/New-York, 2004, p. 23-38, 138139. Tra le discussioni più approfondite delle tesi di Osborne si segnalano: I. MALKIN, Exploring the Validity of the Concept of 'Foundation': a Visit to Megara Hyblaia, in V.B. Gorman - E.W. Robinson (Eds.), Oikistes. Studies in Constitutions, Colonies, and Military Power in the Ancient World Offered in Honor of A.J. Graham, Leiden/Boston/Köln, 2002, p. 196-225; ID., 'Tradition' in Herodotus: The Foundation of Cyrene, in P. DEROW R. PARKER (Eds.), Herodotus and his World. Essays from a Conference in Memory of George Forrest, Oxford, 2003, p. 153-170. Cf. inoltre M. LOMBARDO, Poleis e politeiai nel mondo "coloniale", in S. CATAldi (a cura di), Poleis e Politeiai. Atti del Convegno Internazionale di Storia Greca (Torino, 29 maggio-31 maggio 2002), Alessandria, 2004, p. 355-357. 
sporadica, evidentemente in extremis, di qualche riferimento a lavori usciti nel $2000 \mathrm{o}$ 2001. Il problema, come si evince dalla prefazione, è tutto di carattere editoriale. Non si può dunque fare carico ai singoli autori di una responsabilità che non ricade evidentemente sulle loro spalle (salvo laddove il contributo appare già fuori tempo anche con riferimento agli anni '90) e ci si può solo rammaricare che i loro saggi abbiano dovuto attendere così a lungo la pubblicazione e che l'ambizione del volume di offrire una panoramica ampia e aggiornata possa dirsi realizzata solo per quanto riguarda il primo obbiettivo. Quanto serio sia il problema appare evidente già dal primo capitolo, redatto da M.H. Hansen, che presenta un ottimo studio sull'emporion (Emporion. A Study of the Use and Meaning of the Term in the Archaic and Classical Periods). In una nota (p. 20 n. 97) l'autore precisa di aver consegnato il lavoro prima di poter prendere visione dei risultati delle indagini condotte a Pistiros e pubblicate in $B C H, 123$ (1999) e di non averne quindi potuto tenere conto, se non marginalmente, attraverso l'aggiunta di qualche nota. Lo stesso studioso non ha potuto avvalersi dei risultati delle ricerche più recenti riguardanti il sito di Emporion (Spagna) come risulta da una nota in merito aggiunta dal curatore (p. 8 n. 46). La discussione di Hansen dell'emporion è, ciononostante, eccellente. Con la consueta chiarezza che contraddistingue le sue disamine, lo studioso danese analizza il rapporto tra polis ed emporion, spesso impropriamente contrapposti perché considerati due tipi di insediamento distinti oppure messi talora in relazione, ma non su basi corrette. Come emerge dalla sua accurata lettura delle fonti, la distinzione tra polis ed emporion non è che un costrutto moderno e le due nozioni sono in realtà correlate. Ciò che va tenuto presente è che il termine emporion può avere due valenze: può riferirsi a una parte di una polis (esistevano infatti poleis che avevano un emporion ossia un'area dedicata e istituzioni specificamente finalizzate agli scambi con l'esterno) oppure a un tipo particolare di polis. Le poleis che erano emporia non godevano generalmente di status autonomo bensì erano dipendenti e in quanto sede privilegiata di scambi e di traffici tra Greci e non Greci erano abitate da una popolazione di tipo misto. L'emporion come tipo di comunità sembra affermarsi soprattutto in epoca classica: nell'arcaismo i soli insediamenti di questo tipo sicuramente attestati sono Naucrati ed Emporion (Spagna).

Tra le istallazioni greche d'oltremare vanno annoverati gli insediamenti, temporanei o permanenti, dei Micenei sulle rive del Mediterraneo. Dell'espansione micenea si occupa J. Vanschoonwinkel (Mycenean Expansion) in un capitolo dal taglio alquanto datato. Al problema del mancato aggiornamento, che è comune, come si è detto, anche agli altri saggi, si aggiunge in questo caso un problema di metodo che riguarda l'uso delle fonti. La prima parte del capitolo è infatti dedicata a una rassegna delle testimonianze archeologiche note al momento della stesura del lavoro mentre la seconda consiste in un'esposizione delle leggende inerenti la presenza di eroi greci nel Mediterraneo che vengono sottoposte a un sistematico sondaggio alla ricerca di "reminiscenze", cristallizzate o modificate, da mettere a confronto con i dati archeologici, secondo i canoni di quella che si suole definire "logica combinatoria". Il problema non è, ovviamente, quello di prendere posizione tra credulità o scetticismo in merito alle ipotetiche informazioni desumibili dalle leggende (p. 90-91), magari scegliendo una via intermedia come sembra suggerire l'autore di questo saggio, ma 
quello di distinguere i piani, letterario e archeologico, abbandonando la ricerca di "conferme" immediate tra evidenze di tipo diverso che, in quanto tali, non possono essere rapportate le une alle altre senza prima essere state esaminate criticamente in modo del tutto indipendente. Ciò che occorre soprattutto evitare è di storicizzare $\mathrm{i}$ contenuti delle tradizioni leggendarie mentre vanno tenute presenti le circostanze in cui tali racconti sono stati messi per iscritto e le caratteristiche e gli intenti delle fonti che le hanno trasmesse. Non si può dunque che sottoscrivere ciò che un altro studioso, A.J. Domínguez, osserva più avanti in questo stesso volume, (p. 341): "it is usually futile to seek to establish relationships between Greek myths and legends and archaeological evidence", concetto questo ribadito successivamente dal medesimo autore con riferimento alle tradizioni relative ad eroi dell'epos nella penisola iberica (p. 431). Per quanto riguarda la disamina delle testimonianze archeologiche micenee, a parziale integrazione della rassegna fornita nel saggio di Vanschoonwinkel, va qui segnalato almeno un importantissimo ritrovamento avvenuto in Italia, in località Cisterna di Tolentino nella provincia di Macerata. Si tratta di circa sessanta frammenti di ceramica micenea (coppe, tazze, brocche, giare, alabastra) risalenti al Tardo Elladico III B-III C ${ }^{4}$. L'importanza di questi reperti è eccezionale non solo per la quantità, ma perché si tratta del primo rinvenimento di ceramica micenea in una località relativamente interna delle Marche che si aggiunge ai pochissimi frammenti finora recuperati in territorio marchigiano, in località più prossime alla costa, a Treazzano di Monsampolo, a Montagnolo di Ancona e a Jesi. Queste nuove testimonianze inducono legittimamente a chiedersi se il ruolo del comprensorio marchigiano nella tarda Età del Bronzo non sia stato più rilevante di quanto fin qui ritenuto e se, in particolare, non si debba attribuire a questo territorio la funzione di cerniera tra il sud della penisola, vera area di interazione tra popolazioni locali e trafficanti egei, e l'Italia settentrionale.

Sempre a Vanschoonwinkel si deve il capitolo dedicato alle migrazioni greche in Asia Minore (Greek Migrations to Aegean Anatolia in the Early Dark Age). La distinzione tra questo fenomeno e la colonizzazione arcaica è data qui per scontata e non viene dunque fornita alcuna giustificazione per il trattamento separato dei due movimenti. Tale distinzione è in realtà un costrutto moderno visto che gli autori antichi si rappresentano i due fenomeni negli stessi termini. Negli studi moderni è da tempo invalso l'uso di differenziarli in base al fatto che all'uno - il movimento coloniale - è stato tradizionalmente attribuito un livello di organizzazione non riconosciuto all'altro. Se si conviene però, come ormai da più parti si ammette, che la colonizzazione arcaica è coeva al formarsi della polis (cf. in questo stesso volume E.

4 G. DE MARINIS - P. PALlECCHI - E. PerCOSSI - T. SABBATINI, La ceramica micenea, la pasta vitrea e l'ambra, in Moscosi di Cingoli e Cisterna di Tolentino: due siti dell'età del Bronzo a confronto, Roma, 2003, p. 23-25; M. LuNI, I Greci nel kolpos adriatico, Ankon e Numana, in L. BRACCESI - M. LuNI (a cura di), I Greci in Adriatico, 2, Roma, 2004, p. 15; G.M. FABRINI - G. PACI - R. PERNA (a cura di), Beni archeologici della provincia di Macerata, Macerata, 2004, p. 20, 185. Per altri rinvenimenti recenti di ceramica micenea in Italia si veda D. RIDGWAY, in AR for 2001-2002, p. 118. 
Greco, p. 169) allora la distanza qualitativa tra i due fenomeni si assottiglia ${ }^{5}$. Chi ritiene pertanto opportuno continuare a considerarli separatamente dovrebbe precisare in che cosa consiste la differenza. È solo una distinzione di cronologia? O si ravvisano delle differenze di altro tipo? Per quanto riguarda il contenuto della trattazione, anche in questo caso, come nel capitolo precedente, sono passati in rassegna i dati letterari e quelli archeologici, ma la giustapposizione tra i due tipi di testimonianze è qui meno meccanica e più attenta ai condizionamenti a monte delle tradizioni mitistoriche riguardanti il fenomeno migratorio verso l'Anatolia. Qualche parola andava peraltro spesa su tempi e modi di definizione delle identità etniche in quest'area del mondo greco. Solo alla fine dell' esposizione viene, un po' ellitticamente, affermato che piuttosto che parlare di migrazioni di Eoli, Ioni e Dori, come di fatto avviene in tutto il saggio, sarebbe più opportuno riferirsi a migrazioni in Eolide, Ionia e territorio dorico (p. 136). Tutto resta però implicito senza nemmeno un rimando alle ormai classiche, e chiarificatrici, trattazioni sull'argomento ${ }^{6}$.

All'espansione dei Fenici è dedicato il saggio di H.G. Niemeyer (The Phoenicians in the Mediterranean. Between Expansion and Colonisation: A Non-Greek Model of Overseas Settlement and Presence). A prima vista l'inclusione di un capitolo su questo tema in un'opera dedicata alla colonizzazione greca può apparire singolare, ma lo studioso ne rivendica la legittimità con due motivazioni: la priorità cronologica della mobilità fenicia rispetto alla colonizzazione arcaica, il che implica che $i$ coloni Greci abbiano dovuto fare $i$ conti in alcune aree con presenze fenice, e la diversità dei due modelli di espansione, che suggerisce un esame comparato per meglio evidenziare le rispettive specificità. Il quadro tracciato in questo saggio prevede una prima fase di attività di gruppi di 'mercanti avventurieri' fenici, iniziata dopo la fine dell'Età del Bronzo e durante la quale alcuni trafficanti si sarebbero insediati in territorio straniero dando vita a piccole enclaves o fondachi. Ad essa avrebbe fatto seguito una seconda fase caratterizzata da istallazioni stabili e indipendenti, in pratica un'istituzionalizzazione delle precedenti sedi commerciali, e dunque di segno sostanzialmente diverso rispetto alle apoikiai, che lo studioso considera di carattere prevalentemente agricolo. Ciò sarebbe avvenuto solo a partire dall'VIII secolo in risposta alla crescente presenza dei Greci nel Mediterraneo. A questo secondo stadio viene riportata anche la fondazione di Cartagine, che Niemeyer data alla prima metà dell'VIII secolo, ma che, secondo indagini recenti condotte con il radiocarbonio e di cui anche $G$. Tsetskhladze dà notizia nella sua introduzione (p. XXXV), va retrodatata al IX secolo. Se si accetta questo dato, difficilmente controvertibile, la ricostruzione proposta richiede, almeno per quanto concerne la seconda fase dell'espansione fenicia, una nuova messa a punto.

5 In questo senso si veda ora particolarmente J.M. HALL, A History of the Archaic Greek World ca. 1200-479 BCE, Oxford, 2007, p. 93-118.

6 J.M. HALl, Ethnic Identity in Greek Antiquity, Cambridge, 1997; D. AsHERI, Identità greche, identità greca, in S. SETTIS (a cura di), I Greci. Storia Cultura Arte Società, 2, II, Torino, 1997, p. 14-19. Per gli Eoli, in particolare, si segnala ora A. MELE, Aiolos e gli Aiolodai: tradizioni anatoliche e metropolitane, in A. MELE - M.L. NAPOLITANO A. VisCONTI, Eoli ed Eolide tra madrepatria e colonie, Napoli, 2005, p. 15-24. 
La discussione della colonizzazione greca arcaica entra nel vivo con il capitolo di E. Greco dedicato alla Magna Grecia (Greek Colonisation in Southern Italy: A Methodological Essay). L'esposizione verte in primo luogo sulle origini dei coloni e sulle circostanze che hanno portato alla fondazione delle varie colonie nell'Italia meridionale. Un'altra sezione è dedicata all'organizzazione dello spazio, con puntuali osservazioni sull'urbanistica delle città greche dell'Italia meridionale e sulla definizione delle rispettive chorai, anche in rapporto allo strutturarsi del mondo indigeno. All'importanza del mondo acheo nella regione fa riscontro lo spazio dedicato da Greco a questo ambiente, che è stato oggetto anni addietro di un convegno internazionale i cui Atti sono stati pubblicati a cura dello stesso studioso che non se ne è però potuto evidentemente avvalere per questa trattazione ${ }^{7}$. Segue (ma non dovrebbe, a rigore, precedere?) una sezione, redatta da B. d'Agostino, sulle prime presenze greche in Italia (The First Greeks in Italy). Va precisato che per Italia si intende qui solo la penisola in quanto Sicilia e Sardegna sono oggetto di disamine separate da parte di altri studiosi. La trattazione di d'Agostino è dedicata alle frequentazioni euboiche che hanno interessato la penisola italiana a partire dalla prima metà dell'VIII secolo e ai contatti tra Eubei e popolazioni locali, specialmente dell'Etruria e della Campania. Particolare attenzione è riservata agli adattamenti locali dei modelli greci in campo materiale e culturale, aspetto quest'ultimo ben esemplificato dalla rielaborazione etrusca del simposio, riassorbito in questo ambiente nella sfera funeraria. Il quadro dei rapporti tra Eubei e comunità locali della penisola tracciato in questo saggio è ampio e articolato, anche se la prospettiva oggi si è ulteriormente allargata grazie al lavoro di Laurence Mercuri ${ }^{8}$ che ha mostrato come anche la Calabria meridionale vada inclusa a pieno titolo tra le zone interessate dalle prime frequentazioni euboiche. In una trattazione incentrata sugli Eubei in Occidente non poteva mancare una sezione dedicata a Pithecusa, che d'Agostino considera la prima apoikia, ma di tipo particolare: per l'importanza degli scambi con il mondo tirrenico l'attività di questo insediamento sembra trovare piuttosto rispondenza nel modello che si suole definire, con termine corrente ancorché improprio, "precoloniale" mentre da un punto di vista organizzativo e socio-politico i suoi tratti prefigurano quelli attribuiti alle fondazioni propriamente coloniali. Lo status di Pithecusa resta comunque argomento controverso e la discussione continua ${ }^{9}$

Il capitolo sulla Sardegna, redatto da D. Ridgway (Early Greek Imports in Sardinia), concerne un'area nella quale i Greci non hanno fondato colonie, ma che è stata anch'essa parte di quel circuito esteso di traffici che ha interessato il Mediterraneo nei secoli che hanno preceduto l'età coloniale. Numerosi frammenti di ceramica micenea, rinvenuti prevalentemente a sud del golfo di Oristano, attestano l'interesse

7 E. Greco (a cura di), Gli Achei e l'identità etnica degli Achei d'Occidente. Atti del Convegno Internazionale di Studi (Paestum, 23-25 febbraio 2001), Paestum/Atene, 2002.

8 L. Mercuri, Eubéens en Calabre à l'époque archaïque. Formes de contact et d'implantations, Rome, 2004.

9 Cf. A. Mele, Le anomalie di Pithecusa. Documentazioni archeologiche e tradizioni letterarie, in L'incidenza dell'antico. Dialoghi di Storia greca, 1 (2003), p. 13- 39. 
dei navigatori egei per l'isola, soprattutto per la sua parte meridionale, ${ }^{10}$ e per le sue risorse metallifere mentre $\mathrm{i}$ frammenti di ceramica geometrica euboica ritrovati a Sant'Imbenia, vicino ad Alghero, sono dello stesso tipo di quelli rinvenuti nei corredi funerari dell' Età del Ferro in Etruria. Poco importa se i vettori sono stati gli Eubei stessi $\mathrm{o}$, più probabilmente, i Fenici. Ciò che vale la pena di sottolineare, e lo studioso non manca di evidenziarlo, è che dall'Età del Bronzo in poi il Mediterraneo è stato teatro di un incessante scambio di merci, gestito da genti di diversa origine, e che su questo sfondo di mobilità e di imprenditorialità multietnica si è innestato, secondo logiche e priorità di altro tipo, il movimento coloniale greco di epoca arcaica.

La colonizzazione greca della Sicilia è discussa in modo molto puntuale ed equilibrato da A.J. Domínguez (Greeks in Sicily) che tocca tutti gli aspetti fondamentali del fenomeno: fondazione delle colonie primarie (con rassegna delle principali testimonianze letterarie e archeologiche), loro espansione, subcolonizzazione, sviluppi politici, rapporti con il mondo indigeno sia a livello economico che culturale, con particolare riguardo al riadattamento in ambito locale dei modelli abitativi greci e dell'alfabeto. La sensibilità dello studioso per i processi di interazione greco-indigena gli avrebbe certamente consentito di fare buon uso di quello straordinario monumento scultoreo rinvenuto nel 1999 e noto con il nome di Guerriero di Castiglione ${ }^{11}$, già da altri esaminato in chiave di "ibridismo culturale" 12 , mentre per quanto riguarda specificamente le fondazioni greche la pur vasta bibliografia citata richiede ormai numerose integrazioni: si pensi, per esempio, alla ricca messe di pubblicazioni su Megara Iblea e alla discussione in atto sull'interpretazione del suo primo tracciato urbano ${ }^{13} \mathrm{e}$ ora anche alle prospettive siracusane aperte dalla nuova amplissima trattazione storica, archeologica e bibliografica dedicata alla città ${ }^{14}$.

Il quadro delle presenze greche in Occidente si completa con i capitoli dedicati alla colonizzazione focea e alla frequentazione della penisola iberica, rispettivamente redatti da J.-P. Morel (Phocaean Colonisation) e dallo stesso A.J. Domínguez (Greeks in the Iberian Peninsula). I Focei, come sottolinea Morel, si sono insediati in primo luogo a Lampsaco nell'Ellesponto (615 a.C.?) e poi probabilmente ad Amiso, sulla costa settentrionale dell'attuale Turchia e anche ad Antheia sul Mar Nero, ma ben altrimenti nota e documentata è la loro presenza in Occidente, che lo studioso riesa-

10 Ulteriori ritrovamenti di ceramica micenea non segnalati in questo saggio, avvenuti nell'area di Nora e poco più a nord, presso il Nuraghe Is Baccas, sono notificati dallo stesso RIDGWAY in AR for 2001-2002, p. 118.

11 F. CORDANo - M. Di SAlvatore (a cura di), Il Guerriero di Castiglione di Ragusa, in Hesperia, 16 (2002).

12 T. Hodos, Local Responses to Colonization in the Iron Age Mediterranean, London, 2006, p. 145-147.

13 I. Malkin, Exploring the Validity, cit.; F. DE Angelis, Megara Hyblaia and Selinous: the Development of two Greek City-States in Archaic Sicily, Oxford, 2003; M. GRAS H. Tréziny - H. Broise, Mégara Hyblaea. 5, La ville archaïque: l'espace urbain d'une cité grecque de Sicile orientale, Rome, 2005; A. RoBU, Chronique mégarienne. À propos d'un livre récent portant sur les cités mégariennes de Sicile, in $A C, 75$ (2006), p. 205-212; J.M. HALl, $A$ History, cit., p. 107-110.

14 Siracusa, in BTCGI, 19 (2005), p. 1-386. 
mina soffermandosi soprattutto su Massalia, ma senza trascurare le vicende di Alalia, di Emporion e di Velia (Hyele/Elea). Particolare attenzione è riservata non solo alle singole fondazioni, alla loro espansione, ai loro rapporti non sempre idilliaci con le popolazioni vicine, ma anche alla rete commerciale organizzata dai Focei tra Asia Minore ed estremo Occidente, con epicentro a Focea fino alla caduta della città in mano persiana nel 545 ca. Dopo questa data il quadro muta: dal punto di vista culturale il mondo foceo mantenne una forte coesione e Massalia ne divenne il referente principale, ma dal punto di vista economico ogni città sembra aver proseguito individualmente il proprio cammino. Una caratteristica dell'ambiente foceo coloniale, ben evidenziata in questo contributo, è il suo affiatamento con altri Greci, specie i Calcidesi, ma anche gli Achei. L'intesa soprattutto con i primi era essenziale per consentire ai Focei di avere mano libera nel Tirreno e di contrastare efficacemente le minacce rappresentate da Etruschi e Cartaginesi. Se il saggio di Morel lascia aperta la questione del rapporto cronologico tra frequentazione di Tartesso e fondazione di Massalia ed Emporion (l'accesso foceo all'estremo Occidente precede o segue le due fondazioni?), Domínguez prende decisamente posizione a favore della prima ipotesi. La sua ricostruzione dei rapporti tra Greci e Iberia prevede una fase di intensa frequentazione dell'area di Tartesso da parte di Focea, iniziata sul finire del VII secolo, e la conseguente creazione di punti di appoggio disseminati lungo la costa mediterranea settentrionale. Intorno al 600 Massalia, in Gallia, ed Emporion, in Iberia, si svilupparono come entità politiche mentre altrove nuclei di Focei continuarono a esercitare le loro attività commerciali in centri non greci, coabitando con l'elemento indigeno. Nella seconda metà del V secolo appare in essere in ambito iberico un'intensa attività di scambio tra centri costieri, in cui operavano i Greci, e popolazioni locali alle quali era affidata interamente, secondo lo studioso, la gestione della rete di traffici con l'interno. L'articolata trattazione di Domínguez - corredata, come del resto la sezione da lui curata sulla Sicilia, di molte utili illustrazioni - si sofferma ampiamente sulle ricadute in ambiente locale del contatto con il mondo greco mostrando, in particolare, come già nel corso del VI secolo forme di espressione o di comunicazione quali la scultura e l'alfabeto siano state recepite e rielaborate in ambito indigeno, in funzione delle esigenze dell'élite, partner e referente principale dei trafficanti greci insediati sulla costa.

Dall'estremo Occidente si passa, con la trattazione di J. Boardman, all'Oriente mediterraneo [Greeks in the East Mediterranean (South Anatolia, Syria, Egypt)] che non fu sede di città elleniche, ma che attrasse i Greci per altre ragioni, soprattutto il desiderio di acquisire materie prime, manufatti o uomini. Questo vale anzitutto per Al Mina e per altre località del nord della Siria o della Cilicia. Per quanto riguarda in particolare lo status di Al Mina nella seconda metà dell'vIII secolo viene qui riproposta la tesi che lo studioso ha più volte sostenuto: Al Mina fu un'istallazione euboica, con presenza anche di Ciprioti nell'ultimo quarto del secolo. La prevalenza di ceramica euboica viene infatti considerata riflesso della presenza in loco di coloro che l'hanno prodotta, tesi questa da altri vivacemente contestata. Il problema ha 
suscitato in passato ampio dibattito e la discussione è lungi dall'essere conclusa ${ }^{15}$. Il saggio di J. Boardman esamina poi il prosieguo dei rapporti tra Greci e Oriente mediterraneo, dopo il venir meno degli interessi euboici intorno al 700 ca., e ripercorre quindi la storia dei contatti tra i Greci e l'Egitto, dalle prime fasi fino all'occupazione persiana, con particolare riguardo alle vicende di Naucrati esaminate attraverso le fonti letterarie greche, la documentazione archeologica e le fonti orientali. Chiude il volume una breve sintesi dei risultati della campagna archeologica condotta nel 1999 nel delta dell'Oronte (H. Pamir, Al Mina and Sabuniye in the Orontes Delta: the Sites). L'indagine ha interessato il sito di Al Mina, che non era stato più investigato dai tempi di Sir Leonard Wolley, e ha mostrato che esso era più esteso di quanto indicato dal primo scopritore. Inoltre sono state condotte ricerche che hanno portato all'individuazione della meno nota località di Sabuniye, ultima tappa nella navigazione fluviale dell'Oronte e snodo tra il Mediterraneo e i siti dell'interno. In questa località era stato effettuato un sondaggio da Wolley, ma in seguito il sito era stato trascurato e non se ne conosceva più nemmeno l'esatta localizzazione.

Ciascuno degli studiosi che ha contribuito a questo volume ha esaminato il tema o l'area che gli è stata assegnata fornendo una panoramica sui dati disponibili e le coordinate del dibattito sulle questioni che risultavano aperte o controverse negli ultimi anni del secolo scorso. Le diversità di opinioni che si registrano su taluni problemi (p. es. la questione Tartesso sopra segnalata) sono certamente un arricchimento e non un limite, ma l'assenza di un uso condiviso di un termine basilare come apoikia $^{16}$ e la conseguente diffusa applicazione nei vari saggi della nozione di emporion con una valenza non coerente con quella proposta da Hansen nel volume stesso, ossia perpetuandone la contrapposizione a polis o ad apoikia, senza che tale difformità sia spiegata e giustificata, denotano una carenza di coordinamento che costituisce un limite per un'opera che intende proporsi come manuale (handbook). A questo proposito è ineludibile una domanda alla quale non è facile trovare una risposta: a quale tipologia di lettore è destinata questa pubblicazione? Per lo studente è troppo vasta, oltre che troppo costosa. Per chi fa ricerca può certamente essere vantaggioso disporre di trattazioni sintetiche, esaurienti e di buon livello soprattutto se riguardano aree delle quali non ci si occupa abitualmente o sulle quali si è meno informati, ma il fatto che i vari contributi rispecchino lo stato delle conoscenze di diversi anni addietro ne riduce considerevolmente l'utilità.

15 Cf. J. Bodrdman, Al Mina: the Study of a Site, in AWE 1 (2002), p. 315-331; ID., Al Mina: Notes and Queries, in AWE 4 (2005), p. 278-291; H.G. NIEMEYER, Phoenician or Greek: Is There a Reasonable Way Out of the Al Mina Debate?, in AWE 3 (2004), p. 38-50; ID., There Is No Way Out of the Al Mina Debate, in AWE 4 (2005), p. 292-295; T. HoDOs, op. cit., p. 37-40.

16 Solo per il curatore "insediamento lontano dalla patria" senza particolare e specifica connotazione politica o sociale (p. XLI), per altri polis coloniale nella quale è individuabile una vocazione agricolo-territoriale (p. es. p. 429 e 476-483), per Hansen, invece, è del tutto ingiustificata la tradizionale distinzione tra apoikia, catalogata come polis e ritenuta dedita principalmente all'agricoltura, ed emporion, considerato un'istallazione commerciale non strutturata come polis (p. 32-34). 